\title{
Estrutura populacional de Macrobrachium potiuna (Crustacea, Palaemonidae) no Rio do Moinho, Mangaratiba, Rio de Janeiro, Brasil
}

\author{
Luciana Antunes de $\operatorname{Mattos}^{1}$ \& Lídia Miyako Yoshii Oshiro \\ ${ }^{1}$ Estação de Biologia Marinha, Universidade Federal Rural do Rio de Janeiro - UFRRJ, \\ Rua Sereder, Itacuruçá, CEP 23860-020, Mangaratiba, Rio de Janeiro, RJ, Brasil, e-mail: oshiro@ufrrj.br \\ ${ }^{2}$ Autor para correspondência: Luciana Antunes de Mattos, e-mail: lubiomattos@hotmail.com
}

MATTOS, L.A. \& OSHIRO, L.M.Y. 2009. Population structure of Macrobrachium potiuna (Crustacea, Palaemonidae) in the Moinho's River, Mangaratiba, Rio de Janeiro, Brazil. Biota Neotrop. 9(1): http://www. biotaneotropica.org.br/v9n1/en/abstract?article+bn01309012009.

\begin{abstract}
Samples of Macrobrachium potiuna (Müller, 1880) were collected in the Moinhos' River from July 2001 to June 2002. The aim of this study was providing some information about the population structure of this species. In the laboratory, the shrimps were sexed, measured and classified according to developmental stages: mature, immature and sexually undifferentiated. A total of 1162 shrimps was collected, being 52\% immature, $46 \%$ matures and $2.6 \%$ sexually undifferentiated. The immature individuals showed a positive correlation with the water temperature, occurring with greater frequency until $21^{\circ} \mathrm{C}$. The total length varied from 9.5 to $49.1 \mathrm{~mm}$, with males being the largest ones of the population. The size frequency distribution was characterized by two modes. The sex ratio of 1:1 was observed. A larger number of males was recorded in the spring and ovigerous females in the spring and summer. The juvenile recruitment took place in the autumn, by registering a time lag of four months between the peak of ovigerous females and the entrance of recruits.
\end{abstract}

Keywords: freshwater shrimps, total length, sex ratio, recruitment, Serra do Piloto.

MATTOS, L.A. \& OSHIRO, L.M.Y. 2009. Estrutura populacional de Macrobrachium potiuna (Crustacea, Palaemonidae) no Rio do Moinho, Mangaratiba, Rio de Janeiro, Brasil. Biota Neotrop. 9(1): http://www. biotaneotropica.org.br/v9n1/pt/abstract?article+bn01309012009.

Resumo: Exemplares de Macrobrachium potiuna (Müller, 1880) foram coletados mensalmente no Rio do Moinho, no período de julho/2001 a junho/2002, com o objetivo de fornecer informações sobre a estrutura populacional dessa espécie. No laboratório os camarões foram separados por sexo, mensurados e classificados de acordo com o estágio de desenvolvimento: maturos, imaturos e sexualmente indiferenciados. Foi capturado um total de 1162 indivíduos, sendo 52\% imaturos, 46\% maduros e 2,6\% sexualmente indiferenciados. Os indivíduos imaturos mostraram correlação positiva com a temperatura da água, ocorrendo com maiores frequiências até $21{ }^{\circ} \mathrm{C}$. O comprimento total variou de 9,5 a 49,1 mm, sendo os machos os maiores indivíduos da população. A distribuição de freqüência de tamanho foi caracterizada por duas modas. A razão sexual observada foi de 1:1. Um maior número de machos foi registrado na primavera e de fêmeas ovígeras na primavera e verão. O recrutamento dos juvenis ocorreu durante o outono, registrando-se um atraso de quatro meses entre o pico de fêmeas ovígeras e a entrada dos recrutas.

Palavras-chave: camarões de água doce, comprimento total, razão sexual, recrutamento, Serra do Piloto. 


\section{Introdução}

Avaliações sobre a estrutura populacional contribuem para o conhecimento da biologia e ecologia da espécie no seu ambiente natural, representando uma importante ferramenta no estudo de diferentes grupos, o que possibilita o conhecimento da amplitude de tamanho alcançado pelos indivíduos de uma população (Hartnoll \& Bryant 1990, Begon et al. 1996). Aspectos da estrutura populacional como a razão sexual e a estrutura etária, influenciam a dinâmica populacional devido às variações relacionadas ao sexo e à idade no nascimento e na morte, sendo tais aspectos de suma importância para a compreensão da ocorrência de mudanças populacionais ao longo do tempo. (Ricklefs 1996).

Macrobrachium potiuna (Muller, 1880) é um palemonídeo de pequeno porte, que se distribui nas bacias da vertente Atlântica do Sudeste e Sul do Brasil, desde o Espírito Santo até o Rio Grande do Sul, tendo como habitat preferencial os riachos de montanha, os quais são caracterizados por possuírem correnteza, fundo arenoso e vasta vegetação nas margens (Bond-Buckup \& Buckup 1989, Melo 2003).

Estudos populacionais realizados com M. potiuna abordam não somente a biologia reprodutiva (Bond \& Buckup 1982, Müller \& Carpes 1991, Nazari et al. 2003, Antunes \& Oshiro 2004, Müller et al. 2004) como a estrutura populacional, sendo enfatizados aspectos como distribuição, crescimento, razão sexual e densidade (Souza \& Fontoura 1995, 1996, Souza et al. 1996, Lima \& Oshiro 2000). Todavia poucos são os estudos populacionais sobre essa espécie nos rios do sudeste brasileiro, os quais são constantemente submetidos à ação antrópica. Diante disso, é de grande valia a obtenção de informações bioecológicas sobre a espécie $M$. potiuna, um importante elo da cadeia trófica de ambientes límnicos, que habita áreas montanhosas de Mata Atlântica ameaçadas por pressões antrópicas, especialmente expansão urbana desordenada, atividades turísticas e agrícolas.

O presente estudo se propõe a fornecer informações sobre a estrutura populacional de M. potiuna no Rio do Moinho, verificandose sua distribuição com relação às variáveis ambientais, frequiência sazonal, razão sexual e recrutamento dos juvenis.

\section{Material e Métodos}

O Rio do Moinho ( $22^{\circ} 57^{\prime} 35^{\prime \prime} \mathrm{S}$ e $\left.44^{\circ} 02^{\prime} 26^{\prime \prime} \mathrm{W}\right)$ nasce na Serra do Piloto e é um dos afluentes do principal rio dessa região, o Rio do Saco, que tem sua nascente em altitudes entre 500 e $600 \mathrm{~m}$ e com o nome de Rio da Lapa atravessa a planície que constitui o Saco de Mangaratiba, desembocando no mar após percorrer $11 \mathrm{~km}$ (Semads 2001). O Rio do Saco é um dos poucos cursos d'água cuja bacia é habitada à montante da Rodovia BR 101 e seus afluentes abrigam várias espécies de crustáceos de água doce.

O estudo foi realizado em dois pontos do Rio do Moinho, estando estes eqüidistantes $100 \mathrm{~m}$ e apresentando fluxo de água intenso devido à presença de pequenas quedas d'água. $\mathrm{O}$ primeiro ponto caracterizase por possuir margem circular e leito medindo 4,20 m de diâmetro, sendo o fundo constituído de folhas secas e substrato areno-lodoso, devida à intensa vegetação marginal que sombreia esta área. O segundo ponto localiza-se acima do primeiro e possui aspecto de um córrego, apresentando margem estreita constituída de um lado por arbustos e gramíneas e do outro por rochas, sendo o leito constituído de substrato arenoso, medindo 1,52 m de largura. O mapa da área de estudo encontra-se em Antunes \& Oshiro (2004).

Coletas mensais foram realizadas no período de julho/2001 a junho/2002 por meio de peneiras, com malhas de 0,5 e $3 \mathrm{~mm}$, as quais foram introduzidas sob raízes da vegetação marginal, bem como sob rochas e folhas depositadas no fundo (CPUE, dois coletores durante 10 minutos em cada ponto). Simultaneamente, registrou-se a temperatura da água e a profundidade nos pontos de coleta. Também foram obtidos dados de precipitação acumulada mensal através do programa de monitoramento ambiental da GEO-RIO.

No laboratório, após a identificação e determinação do sexo de cada indivíduo, utilizou-se um paquímetro manual $(0,1 \mathrm{~mm})$ para mensuração do comprimento total (CT) e do comprimento da carapaça (CC). Os indivíduos foram classificados em três grupos quanto ao estágio de desenvolvimento: maduros, imaturos e sexualmente indiferenciados. Foram considerados maduros, em ambos os sexos, aqueles que apresentavam comprimento total igual ou superior ao da menor fêmea ovígera capturada, sendo os demais imaturos. Os indivíduos que não apresentavam caráter sexual secundário foram considerados sexualmente indiferenciados.

Desvios na proporção mensal de indivíduos maduros e imaturos foram averiguados pelo Qui-quadrado $(\alpha=0,05)$, enquanto o índice de correlação de Spearman $(\alpha=0,05)$ foi utilizado na averiguação de associações positivas entre a freqüência de imaturos e maduros com as variáveis ambientais. A normalidade dos dados foi testada com Lilliefors $(\alpha=5 \%)$, utilizando-se o programa BioEstat 2.0 (Aires et al. 2000).

Os espécimes foram distribuídos em classes de tamanho, sendo os desvios entre os sexos averiguados pelo Qui-quadrado $(\alpha=0,05)$, o qual também foi utilizado para averiguar desvios na razão sexual total e mensal. Histogramas sazonais apresentando frequiências de machos, fêmeas ovígeras e não ovígeras nas diversas classes de largura da carapaça foram utilizados para análise da distribuição sazonal. O recrutamento foi analisado pela presença de modas de indivíduos sexualmente indiferenciados pertencentes às menores classes de tamanho. A correlação entre a frequiência mensal de fêmeas ovígeras e a de recrutas foi averiguada através da correlação cruzada, utilizando-se o pacote estatístico PAST (Hammer et al. 2001).

\section{Resultados}

Valores mensais da temperatura da água, profundidade média dos pontos de coleta e precipitação acumulada são apresentados na Figura 1.

Foram analisados 1.162 espécimes de $M$. potiuna, destes $52 \%$ apresentavam-se imaturos, $46 \%$ maduros e $2,6 \%$ sexualmente indi-

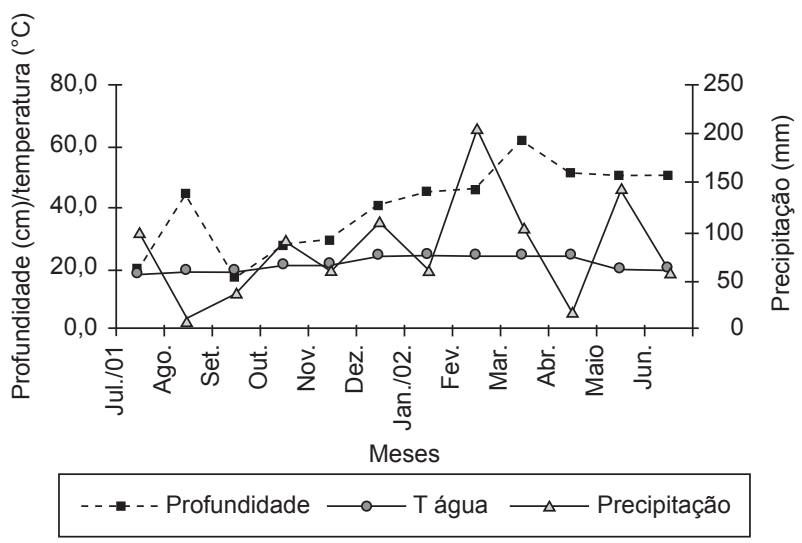

Figura 1. Variação mensal da profundidade, temperatura da água e precipitação acumulada no Rio do Moinho, durante o período de julho/2001 a junho/2002.

Figure 1. Monthly depth variation, water temperature and accumulated rainfall in the Moinho's River, during July/2001 to June /2002. 
ferenciados, ocorrendo um maior número de indivíduos imaturos de agosto a outubro $\left(X^{2}, \mathrm{p}=0.0001, \mathrm{p}=0.0032, \mathrm{p}=0.0000\right)$, em maio $\left(X^{2}, \mathrm{p}=0.0004\right)$ e em junho $\left(X^{2}, \mathrm{p}=0.0001\right)$, meses em que se registraram águas mais frias $(p=0.034)$, entretanto não foram observadas correlações positivas para a precipitação acumulada ( $\mathrm{p}=0.1509)$ e profundidade $(\mathrm{p}=0.7124)$ mensais. Indivíduos maduros ocorreram em maiores proporções em julho $\left(X^{2}, \mathrm{p}=0.0045\right) \mathrm{e}$ janeiro $\left(X^{2}, \mathrm{p}=0.0017\right.$ ) (Figura 2$)$, não sendo registradas associações positivas com as variáveis ambientais testadas $(\mathrm{p}=0.9519, \mathrm{p}=0.7696$, $\mathrm{p}=0.6957$ ).

Os espécimes apresentaram CT variando de 9,5 a 49,1 mm (1,6 a 14,8 mm CC), sendo os machos os maiores indivíduos da população. As amplitudes de tamanho registradas para cada categoria demográfica encontram-se na Tabela 1.

Os espécimes foram distribuídos em 15 classes de CT com amplitude de $3 \mathrm{~mm}$, de acordo com as categorias demográficas acima mencionadas. A distribuição não normal da população (Lilliefors; $\mathrm{p}=0,0434)$, evidenciou bimodalidade em ambos os sexos, ocorrendo nas duas primeiras classes presença marcante de indivíduos

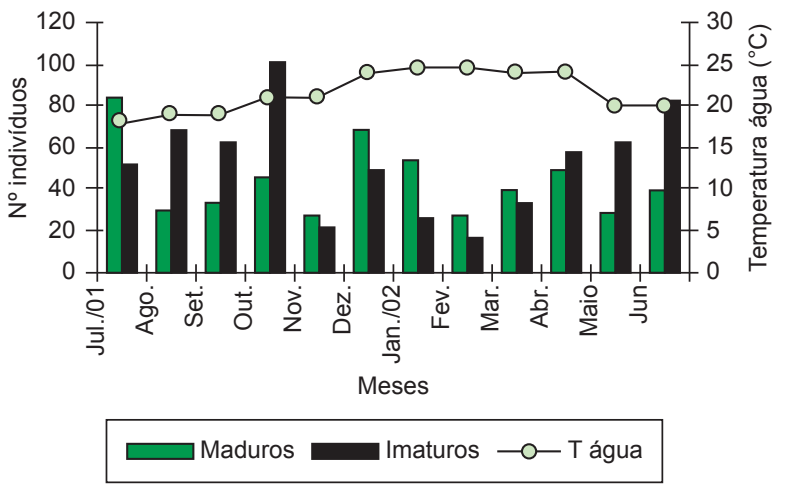

Figura 2. Freqüência mensal de indivíduos maduros e imaturos de $M$. potiuna de acordo com a temperatura da água no Rio do Moinho, durante o período de julho/2001 a junho/2002.

Figure 2. Macrobrachium potiuna immature and mature individuals monthly frequency according to the water temperature in the Moinho's River, during July/2001 to June /2002.

Tabela 1. Amplitude de tamanho $(\mathrm{CT})$ e número de espécimes $(\mathrm{N})$ registrado para cada categoria demográfica $(\mathrm{MI}=$ machos imaturos, $\mathrm{MM}=$ machos maduros, $\mathrm{FI}=$ fêmeas imaturas, $\mathrm{FM}=$ fêmeas maduras e $\mathrm{SI}=$ sexualmente indiferenciados) de M. potiuna durante o período de julho/2001 a junho/2002 no Rio do Moinho.

Table 1. Macrobrachium potiuna size range and number of specimens $(\mathrm{N})$ for each demographic category $(\mathrm{MI}=$ immature males, $\mathrm{MM}=$ mature males, $\mathrm{FI}=$ immature females, $\mathrm{FM}=$ mature females, $\mathrm{FOV}=$ ovigerous females, SI = undifferentiated sexually), during July/2001 to June/2002 in the Moinho's River.

\begin{tabular}{lcr}
\hline Categorias demográficas & $\begin{array}{c}\text { Amplitude de tamanho } \\
(\mathbf{m m})\end{array}$ & $\mathbf{N}$ \\
\hline MI & $14,7 \dashv 25,8$ & 327 \\
MM & $25,9-49,1$ & 222 \\
FI & $14,8-25,8$ & 275 \\
FM & $26,0-39,5$ & 205 \\
FOV & $25,9-40,3$ & 102 \\
SI & $9,5 \dashv 14,5$ & 31 \\
\hline
\end{tabular}

sexualmente indiferenciados. A presença de indivíduos imaturos foi observada entre 14,1 e $26,0 \mathrm{~mm}$, obtendo-se no início desse intervalo um predomínio significativo de fêmeas, e no final de machos $\left(X^{2}, \mathrm{p}=0,0000\right)$. Classes intermediárias e finais foram marcadas pela ocorrência de indivíduos maduros, registrando-se um predomínio de fêmeas maduras de 29,1 até os 35,0 mm $\left(X^{2}, \mathrm{p}=0.0001\right)$ (Figura 3).

A razão sexual observada para a população não diferiu do esperado $1: 1\left(X^{2}=14.5 ; \mathrm{GL}=11 ; \mathrm{p}=0,1592\right)$. Mensalmente, observaram-se desvios não significativos na proporção de machos entre setembro e dezembro, coincidindo com o início do período reprodutivo, e de fêmeas entre janeiro e junho (Tabela 2).

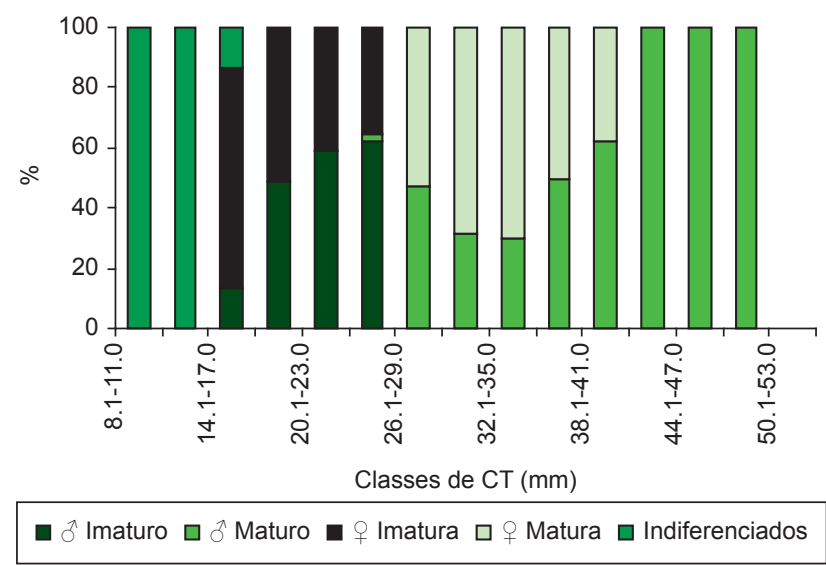

Figura 3. Distribuição de freqüência das categorias demográficas de M. potiuna, no Rio do Moinho, em classes de comprimento total, durante o período de julho/2001 a junho/2002. (*) Diferença significativa entre os sexos obtida pelo teste $X^{2}$.

Figure 3. Macrobrachium potiuna frequency distribution of demographic categories in the Moinho's River in total length classes, during July/2001 to June 12002. (*) Significant difference between the sexes obtained by the $X^{2}$ test.

Tabela 2. Frequiência absoluta e teste do Qui-quadrado $\left(X^{2}\right)$ entre machos e fêmeas de M. potiuna durante o período de julho/2001 a junho/2002 no Rio do Moinho. ${ }^{*} \mathrm{p}<0,05=$ diferença significativa entre os sexos.

Table 2. Macrobrachium potiuna absolute frequency and the Chi-square test $\left(X^{2}\right)$ between males and females, during July/2001 to June/2002 in the Moinho's River. *p $<0,05=$ significant difference between the sexes.

\begin{tabular}{lccc}
\hline \multicolumn{1}{c}{ Mês } & Machos & Fêmeas & $X^{2}(*)$ \\
\hline Julho/01 & 68 & 67 & $\mathrm{p}=0,9314$ \\
Agosto & 49 & 49 & $\mathrm{p}=1,0000$ \\
Setembro & 52 & 44 & $\mathrm{p}=0,4142$ \\
Outubro & 81 & 65 & $\mathrm{p}=0,1854$ \\
Novembro & 26 & 23 & $\mathrm{p}=0,6682$ \\
Dezembro & 62 & 55 & $\mathrm{p}=0,5175$ \\
Janeiro/02 & 31 & 45 & $\mathrm{p}=0,1083$ \\
Fevereiro & 16 & 29 & $\mathrm{p}=0,0526$ \\
Março & 29 & 41 & $\mathrm{p}=0,1515$ \\
Abril & 43 & 52 & $\mathrm{p}=0,3558$ \\
Maio & 40 & 45 & $\mathrm{p}=0,5876$ \\
Junho & 52 & 67 & $\mathrm{p}=0,1691$ \\
Total & 459 & 582 & $\mathrm{p}=0,1592$ \\
\hline
\end{tabular}


O recrutamento dos juvenis teve início no verão, porém no outono obteve-se o pico desse recrutamento, enquanto a atividade reprodutiva foi registrada no verão e na primavera, através das modas de fêmeas ovígeras (Figura 4). A correlação cruzada indicou um período de atraso correspondente a quatro meses entre a freqüência mensal de fêmeas ovígeras e a de recrutas (Figura 5).

\section{Discussão}

Durante o período de coleta, os meses de janeiro e fevereiro mostraram-se os mais quentes, registrando-se $24,5{ }^{\circ} \mathrm{C}$, e julho o mês mais frio, com $18^{\circ} \mathrm{C}$. No que se refere à precipitação, fevereiro mostrou-se o mês mais chuvoso, com 205,2 mm, e agosto, com precipitação acumulada de apenas $10 \mathrm{~mm}$, o mais seco, o que pode ter refletido, respectivamente, na maior profundidade do ambiente límnico registrada em março e na menor em setembro.

Um maior número de indivíduos imaturos na população pode ser explicado por erros de amostragem ou pela seleção de habitats ao longo do rio, sendo os camarões adultos favorecidos por uma maior quantidade de refúgios na margem do rio. A ocupação diferencial de habitats também foi reportada por Darnell (1956) e Galvão \& Bueno (1999) em estudos com Atya scabra (Leach, 1815), por Fransozo et al. (2004) com Macrobrachium iheringi (Ortmann, 1897) e por Lima et al. (2006) para os atídeos Potimirim glabra (Kingsley, 1878) e Potimirim potimirim (Müller, 1881).
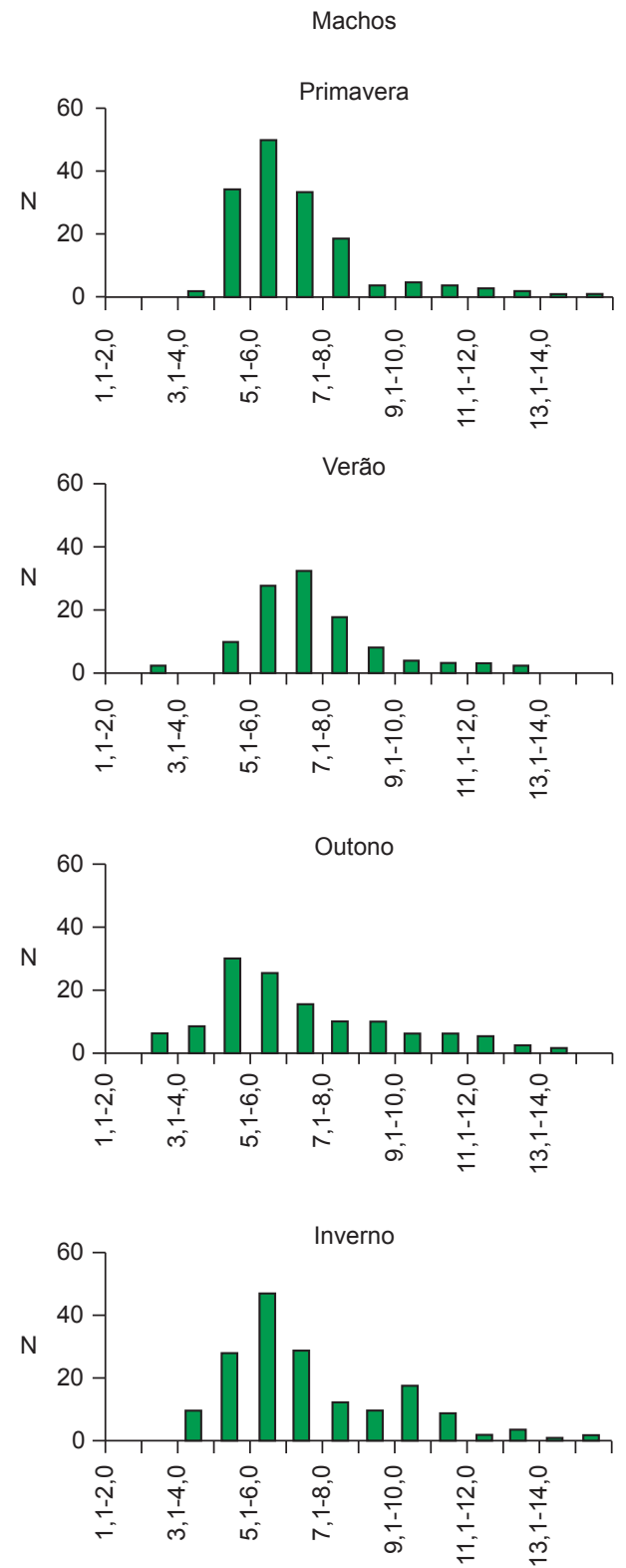
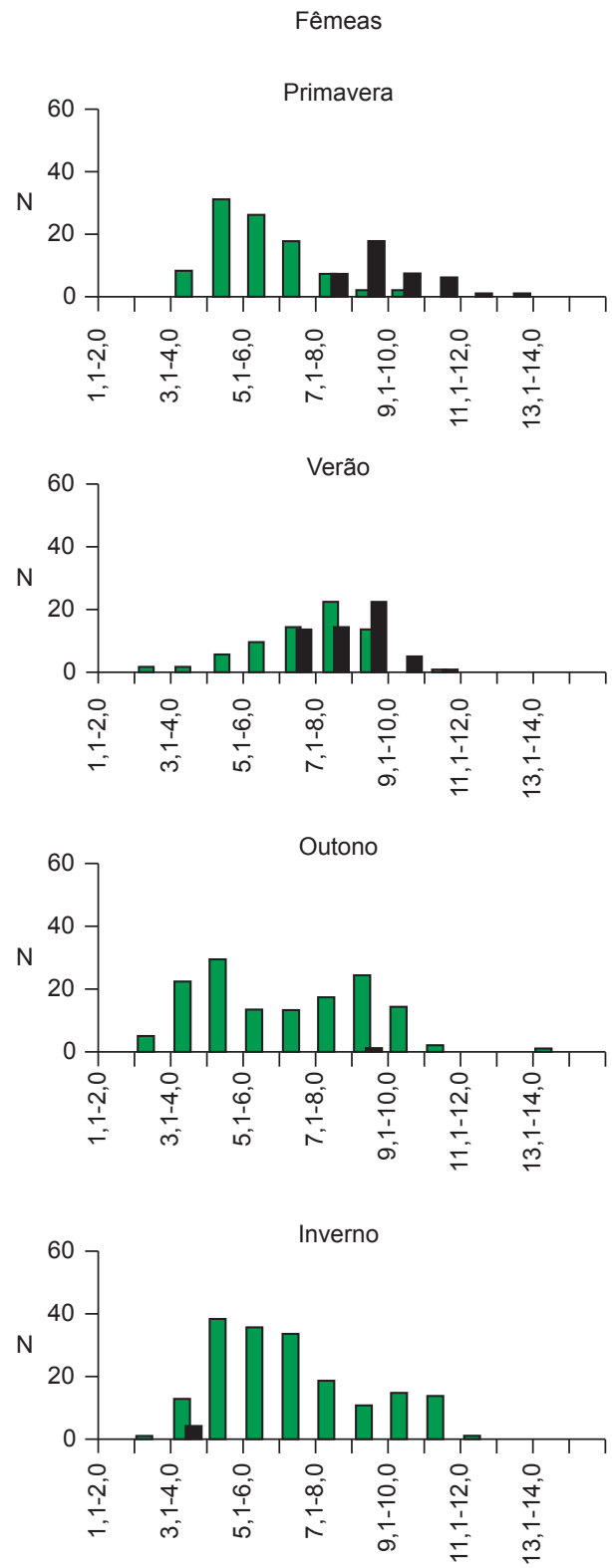

Classes de CC $(\mathrm{mm})$

Figura 4. Distribuições de freqüências sazonais do comprimento da carapaça (CC) de machos, fêmeas não ovígeras (F) e fêmeas ovígeras (Fov) de M. potiuna, no Rio do Moinho, durante o período de julho/2001 a junho/2002.

Figure 4. Macrobrachium potiuna seasonal frequency distribution of the carapace length (CC) of males, females (F) and ovigerous females (Fov) in the Moinho's River, during July/2001 to June/2002. 


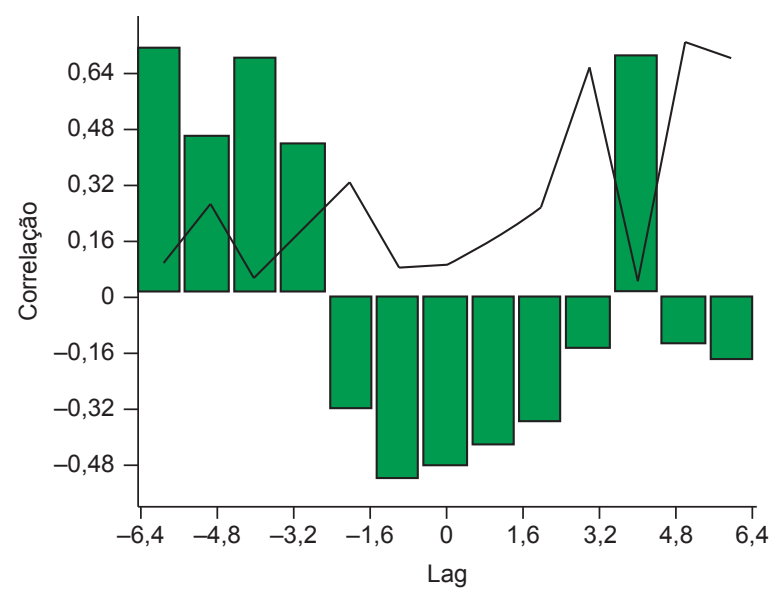

Figura 5. Análise de correlação cruzada entre a freqüência mensal de fêmeas ovígeras e a de recrutas no Rio do Moinho, durante o período de julho/2001 a junho/2002. Linha representa o valor de $\mathrm{p}(\alpha=0,05)$.

Figure 5. Cross correlation analysis between the monthly frequency of the ovigerous females and the recruits in the Moinho's River, during July/2001 to June/ 2002. Line is the $p$ values $(\alpha=0.05)$.

Um predomínio de indivíduos imaturos nos meses em que a temperatura da água não ultrapassou $21^{\circ} \mathrm{C}$ sugere a íntima relação entre esse fator abiótico e o crescimento desses indivíduos. Assim, águas mais frias apresentam maiores níveis de oxigenação e podem favorecer maiores taxas metabólicas, propiciando um melhor desenvolvimento desses organismos. Todavia temperaturas muito baixas podem reduzir as taxas de desenvolvimento em crustáceos, como em Macrobrachium rosenbergii (De Man, 1879), palemonídeo que migra para áreas de menor profundidade, onde emergem a porção dorsal do corpo a fim de minimizar os efeitos de temperaturas inferiores a $15^{\circ} \mathrm{C}$ (Cavalcante et al. 1986).

A variação de tamanho apresentada na população estudada, bem como para aquela registrada em afluentes do Rio Paraíba do Sul, em que o tamanho máximo foi de 45,6 mm (Lima \& Oshiro 2000), sugere a presença de menores indivíduos para a região sudeste em relação às populações encontradas no sul do Brasil, onde foram capturados exemplares de até $54 \mathrm{~mm}$ (Müller et al. 1999, Bond-Buckup \& Buckup 1989). Portanto, pode-se inferir que tais diferenças decorram possivelmente das distintas condições climáticas e de latitude entre tais regiões. Segundo Hartnoll (1982), o tamanho mínimo atingido pelos animais bem como a taxa de crescimento são afetados pelas condições ambientais a que estes estão submetidos, sobretudo a disponibilidade de alimento e temperatura.

A seleção natural pode favorecer machos maiores se esses apresentam vantagens sobre os demais, seja na competição por fêmeas receptivas ou mesmo para maximizar as chances de cópulas bem sucedidas (Abrams 1988). De acordo com Mauchline (1977), o crescimento entre os sexos mostra-se similar até a maturidade, podendo a partir daí tornar-se mais lento em fêmeas, devido a um prolongamento do período de intermuda e um menor incremento em comprimento, o que está relacionado a um maior investimento energético para processos de produção e incubação dos ovos.

A ocorrência de indivíduos sexualmente indiferenciados e imaturos nas classes de tamanho inferiores e a marcada presença dos maduros a partir das classes intermediárias sugerem que o crescimento populacional é contínuo, enquanto a bimodalidade observada em ambos os sexos indica a presença de sazonalidade nos eventos reprodutivos e de recrutamento (Spivak et al. 1991, Tsuchida \& Watanabe 1997). A observação de um acentuado desvio na proporção de fêmeas na terceira classe de tamanho pode ser resultante de erros na sexagem, tendo sido considerados como fêmeas, os machos pequenos que não apresentavam caráter sexual secundário.

A estabilidade populacional conferida pela ausência de desvios entre os sexos foi observada não só no presente estudo, como também por Lima \& Oshiro (2000) nos afluentes do Rio Paraíba do Sul. Já na região sul, foram observados desvios para fêmeas (Santa Catarina) e machos (Rio Grande do Sul), registrados respectivamente, por Müller \& Carpes (1991) e Souza \& Fontoura (1996). Segundo esses últimos autores, estes resultados podem refletir diferentes condições ambientais e de predação, que atuam de forma distinta na mortalidade de cada sexo. Não somente as condições ambientais e bióticas (muda, migração, predação, etc.) podem levar a ocorrência de desvios a favor de um dos sexos, como também os processos de seleção.

A obtenção de uma menor proporção de fêmeas, durante o início do período reprodutivo está possivelmente associada ao fato de que as formas ovígeras refugiam-se em determinados locais, na tentativa de tornarem-se menos susceptíveis aos predadores. Segundo Howard \& Lowe (1984), a predação sobre fêmeas maduras e ovígeras pode ser explicada não só pelo fato dessas atingirem tamanhos maiores que o dos machos, como por fatores que interferem nos mecanismos de escape das fêmeas ovígeras, como a massa e o volume dos ovos, tornando-as mais pesadas e prejudicando a rápida flexão do abdome. Assim, na Austrália, uma espécie de ave da ordem Ciconiiformes, o Colhereiro-real (Platalea regia), é o maior predador de Macrobrachium intermedium (Stimpson, 1860), ocorrendo maior intensidade de predação sobre fêmeas maduras e ovígeras.

Uma maior abundância de fêmeas ovígeras no verão e na primavera caracteriza a ocorrência do período reprodutivo da espécie. Por outro lado, a presença de um maior número de machos na primavera, sugere que tais indivíduos tornam-se mais susceptíveis à captura, enquanto estão à procura de fêmeas.

Apesar da maior freqüência de juvenis ter sido verificada durante o outono, ocorreram no verão os primeiros registros desse recrutamento. Bond \& Buckup (1982) investigaram o período de postura e eclosão desta espécie no Rio Grande do Sul e verificaram que este pode levar de três a quatro meses, iniciando a postura em setembro e a eclosão das últimas larvas em dezembro ou janeiro. Neste caso, assim como no presente estudo, é esperada uma marcada entrada de juvenis na população com a chegada do outono. O padrão de recrutamento observado durante o outono ocorre devido à intensa atividade reprodutiva das fêmeas em meses anteriores (verão). Assim, uma maior freqüência em abril está associada ao pico de fêmeas ovígeras em janeiro, podendo sugerir que no Rio do Moinho a espécie atinge 9,5-14,5 mm de CT (2,3 a 3,5 mm de CC) em três meses.

Uma maior entrada de juvenis no outono e reduzida entrada no verão, embora se tenha registrado nessa estação o pico de fêmeas ovígeras, provavelmente pode ser explicada pela ação da temperatura no desenvolvimento e maturação dos tecidos gonadais (Adiyody \& Adiyody 1970, Wear 1974), como também por sua ação catalítica sobre as diferentes fases larvais (Boolootian et al. 1959). Segundo Emmerson (1994) uma estratégia reprodutiva comum entre os crustáceos consiste em realizar a postura durante o verão, quando se registram temperaturas mais elevadas e abundância de fitoplâncton, facilitando o desenvolvimento dos novos indivíduos. Tal fato explica um atraso de quatro meses entre a freqüência de fêmeas ovígeras e a de recrutas do presente estudo, os quais são resultantes das desovas ocorridas no início de janeiro. Um atraso similar foi reportado para $M$. iheringi em um riacho de Botucatu (SP), ocorrendo as primeiras formas ovígeras no verão e os recrutas no outono (Fransozo et al. 2004)

Nossos dados mostram que a população de $M$. potiuna é caracterizada pela ocorrência de sazonalidade nos eventos reprodutivos e de recrutamento, sendo a temperatura da água um fator relevante 
para a liberação e desenvolvimento das formas larvais. Considerando que atividades antrópicas são as principais causas das variações das características físicas e químicas de ambientes límnicos, é de suma importância a manutenção de riachos montanhosos, visto que os fenômenos ocorrentes nesses ecossistemas determinam alterações no ciclo biológico de diversas espécies que os utilizam, como é o caso de M. potiuna.

\section{Agradecimentos}

A CAPES pelo apoio financeiro, aos Drs. Nelson F. Fontoura e Sérgio L. S. Bueno pelas valiosas sugestões fornecidas à dissertação apresentada ao Programa de Pós-Graduação em Biologia Animal da UFRRJ e aos Srs. Vanderlei Antunes e Danilo Mattos pelo auxílio nas coletas mensais.

\section{Referências Bibliográficas}

ABRAMS, P.A. 1988. Sexual difference in resource use in hermit crabs: consequences and causes. In Behavioral adaptation to intertidal life (G. Chelazzi \& M. Vannini, eds.). Plenum, New York, p. 283-296.

ADIYODY, K.G. \& ADIYODY, R.G. 1970. Endocrine control of reproduction in decapoda crustacean. Biol. rev. 45:121-165.

ANTUNES, L.S. \& OSHIRO, L.M.Y. 2004. Aspectos reprodutivos do camarão de água doce Macrobrachium potiuna (Müller) (Crustacea, Decapoda, Palaemonidae) na Serra do Piloto, Mangaratiba, Rio de Janeiro, Brasil. Revta. Bras. Zool. 21(2):261-266.

AYRES, M., AYRES Jr., M., AYRES, D.L \& SANTOS, A.S. 2000. BioEstat 2.0: aplicações estatísticas nas áreas das ciências biológicas e médicas. Sociedade Civil Mamirauá; CNPq, Belém, Brasília.

BEGON, M., MORTIMER, M. \& THOMPSON, D.J. 1996. Population ecology: a unified study of animals and plants. 3 ed. Blackwell Science, London.

BOND, G. \& BUCKUP, L. 1982. O ciclo reprodutor de Macrobrachium borelli (Nobili,1896) e Macrobrachium potiuna (Muller, 1880) (Crustacea, Decapoda, Palaemonidae) e suas relações com a temperatura. Revta. Bras. Biol. 42(3):473-483.

BOND-BUCKUP, G. \& BUCKUP, L. 1989. Os Palaemonidae de águas continentais do Brasil meridional (Crustacea, Decapoda). Revta. Bras. Biol. 49(4):883-896.

BOOlOOTIAN, R.A.A., GIESE, A.C., FARMANFARMAIN, A. \& TUCKER, J. 1959. Reproductive cycles of five west coast crabs. Physiol. Zool. 32:213-220.

CAVALCANTE, L.B., CORREA, E.S. \& CORDEIRO, E.A. 1986. Camarão: manual de cultivo do Macrobrachium rosenbergii (pitu havaiano - gigante da Malásia). Aquac. res. 21:375-385.

DARNELL, R.M. 1956. Analysis of a population of the tropical freshwater shrimp Atya scabra (Leach). Am. midl. nat. 55:131-138.

EMMERSON, W.D. 1994. Seasonal breeding cycles and sex ratio of eight species of crabs from Magazana, a mangrove estuary in Transkei, Southern Africa. J. crustac. Biol. 14(3):568-578.

FRANSOZO, A., RODRIGUES, F.D., FREIRE, F.A.M. \& COSTA, R.C. 2004. Reproductive biology of the freshwater prawn Macrobrachium iheringi (Ortamann, 1897) (Decapoda: Caridae: Palaemonidae) in the Botucatu region, São Paulo, Brazil. Nauplius, 12(2):119-126.

GALVÃO, R. \& BUENO, S.L.S. 1999. Population structure and reproductive biology of the camacuto shrimp, Atya scabra (Decapoda: Caridea: Atyidae) from São Sebastião, Brazil. In The biodiversity crisis and crustacea (J.C.V.V, Klein \& F.R. Schram, eds.). Crustacean Issues, Rotterdam, p. 291-299.

HAMMER, O., HARPER, D.A.T. \& RYAN, P.D. 2001. Past: paleontological statistics software package for education and data analysis. Paleontologia Electronica 4(1):1-9.

HARTNOLL, R.G. \& BRYANT, A.D. 1990. Size-frequency distributions in Decapod Crustacea: the quick, the dead, and the cast-offs. J. crustac. biol. 10(1):14-19.
HARTNOLL, R.G. 1982. Growth. In The biology of Crustacea (L.G. Abele, ed.). Academic Press, New York, p. 111-196.

HOWARD, R.K. \& LOWE, K.W. 1984. Predation by birds as a factor influencing the demography of an intertidal shrimp. J. exp. mar. biol. Ecol. 74:53-66.

LIMA, E.A.C. \& OSHIRO, L.M.Y. 2000. Distribuição, abundância e biologia reprodutiva de Macrobrachium potiuna (Müller, 1880) (Crustacea, Decapoda, Palaemonidae) do Rio Paraíba do Sul, RJ. Acta biol. Leopoldensia, 22(1):67-77.

LIMA, G.V., SILVEIRA, C.M. \& OSHIRO, L.M.Y. 2006. Estrutura populacional dos camarões simpátricos Potimirim glabra e Potimirim potimirim (Crustácea, Decapoda, Atyidae) no rio Sahy, Rio de Janeiro, Brasil. Iheringia, Ser. Zool. 96(1):81-87.

MAUCHLINE, I. 1977. Growth of shrimps, crabs and lobsters: an assessment. J. Cons. Int. Explor. Mer. 37(2):162-169.

MELO, G.A.S. 2003. Manual de identificação dos Crustacea Decapoda de água doce do Brasil. Universidade de São Paulo, São Paulo.

MÜLLER, Y.M.R. \& CARPES, S. 1991. Macrobrachium potiuna (Müller): aspectos do ciclo reprodutivo e sua relação com parâmetros ambientais (Crustacea, Decapoda, Palaemonidae). Revta. Bras. Zool. 8:23-30.

MÜLLER, Y.M.R., AMMAR, D. \& NAZARI, E.M. 2004. Embryonic development of four species of palaemonid prawns (Crustacea, Decapoda): pre-naupliar, naupliar and post-naupliar periods. Revta. Bras. Zool. 21(1):27-32.

MÜLLER, Y.M.R., NAZARI, E.M., AMMAR, D., FERREIRA, E.C., BELTRAME, I.T. \& PACHECO, C. 1999. Biologia dos Palaemonidae (Crustacea, Decapoda) da bacia hidrográfica de Ratones, Florianópolis, Santa Catarina, Brasil. Revta. Bras. Zool. 16(3):629-636.

NAZARI, E.M., SIMÕES-COSTA, M.S., MULLER, Y.M.R., AMMAR, D. \& DIAS, M. 2003. Comparisons of fecundity, egg size, and egg mass volume of the freshwater prawns Macrobrachium potiuna e Macrobrachium olfersi (Decapoda, Palaemonidae). J. crustac. Biol. 23(4):862-868.

RICKLEFS, R. 1996. A economia da natureza. 3 ed. Guanabara Koogan, Rio de Janeiro.

Secretaria de Estado de Meio Ambiente e Desenvolvimento Sustentável. 2001. Bacias hidrográficas e recursos hídricos da macroregião ambiental. 2. Bacia da Baía de Sepetiba. Rio de Janeiro: SEMADS.

SOUZA, G.D. \& FONTOURA, N.F. 1995. Crescimento de Macrobrachium potiuna no Arroio Sapucaia, Município de Gravataí, RS (Crustacea, Decapoda, Palaemonidae). Rio Grande do Sul. Revta. Bras. Biol. 55(1):51-63.

SOUZA, G.D. \& FONTOURA, N.F. 1996. Reprodução, longevidade e razão sexual de Macrobrachium potiuna (Müller,1880) (Crustacea, Decapoda, Palaemonidae) no Arroio Sapucaia, Município de Gravataí, Rio Grande do Sul. Nauplius, 4:49-60.

SOUZA, G.D., BARROS, M.P. \& BRAUN, A.S. 1996. Densidade populacional de Macrobrachium potiuna (Müller, 1880) (Decapoda, Palaemonidae) no Arroio Sapucaia, Localidade Morro Agudo, Município de Gravataí, RS. Nauplius, 4:61-72.

SPIVAK, E.D., GAVIO, M.A. \& NAVARRO, C.E. 1991. Life history and structure of the Word's Southernmost Uca population: Uca uruguayensis (Crustacea, Brachyura) in Mar Chiquita lagoon (Argentina). Bull. mar. Sci 48(3):679-688.

TSUCHIDA, S. \& WATANABE, S. 1997. Growth and reproduction of the grapsid crab Plagusia dentipes (Decapoda, Brachyura). J. crustac. Biol. 17(1):90-97.

WEAR, R.G. 1974. Incubation in British decapod Crustacea, and the effects of the temperature on the rate and success of embryonic development. J. Mar. Biol. Assoc. U.K. 54:745-762. 